11

\title{
Нуклеация и рост зародышей новой фазы в гетерогенных реакциях
}

\author{
(C) Н.М. Корценштейн
}

Энергетический институт им. Г.М. Кржижановского, Москва, Россия

E-mail: naumkor@yandex.ru

Поступила в Редакцию 16 июля 2019 г.

В окончательной редакции 16 июля 2019 г.

Принята к публикации 25 июля 2019 г.

Получены выражения для скорости нуклеации и скорости роста конденсированных частиц в гетерогенной реакции определенного вида, что позволит описать кинетику процесса образования конденсированной фазы в некоторых сложных системах. Использованы методы классической теории нуклеации, а также предположение о механизме протекания реакции.

Ключевые слова: простые и сложные системы, гетерогенные реакции, нуклеация, рост зародышей.

DOI: 10.21883/FTT.2019.12.48565.02ks

1. С позиций химической термодинамики процесс конденсации в простых системах (например, водяной парвода) можно рассматривать как гетерогенную реакцию вида

$$
C \rightarrow C^{*} \text {. }
$$

Уже в изначально двухкомпонентной системе возникает несколько возможных путей процесса конденсации

$$
\begin{gathered}
A+B \rightarrow(A+B)_{\text {sol }}, \\
\left.\begin{array}{c}
A+B \rightarrow C \\
C \rightarrow C^{*}
\end{array}\right\}, \\
A+B \rightarrow C^{*} .
\end{gathered}
$$

Случай (2) соответствует образованию в результате конденсации раствора двух нереагирующих компонентов. Изучение этого процесса с точки зрения теории нуклеации начато в работе [1] и продолжено многими авторами впоследствии. В [2] была впервые рассмотрена многокомпонентная нуклеация при образовании многокомпонентного раствора. Результаты серии работ, посвященных многокомпонентной нуклеации при распаде многокомпонентного твердого раствора, суммированы в монографии [3].

Случай (3) соответствует реагирующим компонентам газовой фазы. Соответственно процесс конденсации в сложных многокомпонентных реагирующих системах согласно (3) связан с цепочкой газофазных реакций, ведущих к образованию пара и конденсированной фазы. При этом молекулярные составы пара и конденсированной фазы одинаковы. Процессы типа (3) реализуются в природных явлениях, лабораторных исследованиях и технологических процессах (см., например, [4-6]).

Случай (4) соответствует гетерогенному реагированию молекул газовой фазы на поверхности зародышей с образованием молекул конденсированной фазы. При этом в газовой фазе отсутствуют как мономерные, так и полимерные молекулы пара, соответствующего $C^{*}-C, C_{2}, \ldots C_{n}$. Такая ситуация реализуется, повидимому, в системе $\mathrm{Al}-\mathrm{O}$, где конденсированная фаза представлена оксидом $\mathrm{Al}_{2} \mathrm{O}_{3}$, а газовая фаза представлена молекулами $\mathrm{Al}_{2} \mathrm{O}, \mathrm{AlO}, \mathrm{AlO}_{2}$ и атомами Al. Процесс, аналогичный (4), реализуется при твердофазной гетероэпитаксии карбида кремния на кремниевой подложке [7]. Отметим, что конденсация в гетерогенной реакции может рассматриваться как обратный процесс по отношению к неконгруэнтному испарению [8]. Именно процесс конденсации в соответствии с (4) был предметом рассмотрения в данной работе.

2. По аналогии с классической теории нуклеации (см. [9]) в рассматриваемом случае (4) рост зародыша определяется реакциями вида

$$
Z_{g-1}+A+B=\underset{k_{g}^{\prime}}{\stackrel{k_{g-1}}{\rightleftarrows}} Z_{g}, \quad 1 \leq g \leq G,
$$

где $Z_{g}$ - зародыш из $g$ молекул $C^{*}$. Баланс числа зародышей, образующихся и распадающихся в двух последовательных реакциях (5), приводит к кинетическому уравнению, совпадающему по виду с аналогичным уравнением в случае простой системы

$$
\frac{\partial N_{g}}{\partial t}=J_{g}-J_{g+1} \text {. }
$$

Здесь $J$ - скорости реакций (5). Будем рассматривать стационарную нуклеацию, т.е. $\partial N_{g} / \partial t=0$. При этом условии согласно уравнению (7) имеет место стационарный поток зародышей в пространстве их размеров

$$
J_{g-1}=J_{g}=\ldots=J_{G}=I,
$$

для которого, следуя подходу Я.Б. Зельдовича [9], получаем выражение для скорости стационарной нуклеации

$$
I=\left(\int_{1}^{G} d g / k(g) N^{\prime}(g) N_{A} N_{B}\right)^{-1} .
$$


Вычисление скорости стационарной нуклеации по (8) предполагает знание явного вида функций $k(g)$ и $N^{0}(g)$.

3. На основании „квазихимического“ подхода Я.И. Френкеля $[10]$ из условия термодинамического равновесия в суммарной реакции образования зародышей из $g$ молекул $C^{*}$

$$
g(A+B) \rightarrow Z_{g}
$$

$$
g\left[\mu_{A}\left(p_{A}, T\right)+\mu_{B}\left(p_{B}, T\right)\right]=\mu_{Z_{g}}^{0}+k T \ln \frac{N_{g}^{0}}{N_{\Sigma}},
$$

равновесное распределение зародышей можно записать в виде (см. также [3,11])

$$
\begin{aligned}
N_{g}^{0} & =N_{\Sigma} \exp \left[-\left(\mu_{Z_{g}}^{0}-g\left(\mu_{A}\left(p_{A}, T\right)+\mu_{B}\left(p_{B}, T\right)\right)\right) / k T\right] \\
& \equiv N_{\Sigma} \exp [-\Delta \Phi(g) / k T] .
\end{aligned}
$$

Здесь $N_{\Sigma}-$ полное число частиц (молекул, зародышей и комплексов).

Использование жидкокапельной аппроксимации для химического потенциала зародыша $\mu_{Z_{g}}^{0}$

$$
\mu_{Z_{g}}^{0}=g \mu_{C^{*}}+\chi \sigma g^{2 / 3},
$$

позволяет записать выражения, определяющие работу образования зародыша и его критический размер, в виде, формально совпадающем с соответствующими выражениями для простой системы [10]

$$
\begin{gathered}
\Delta \Phi(g)=-g k T \ln \tilde{s}+\chi \sigma g^{2 / 3}, \\
g_{c r}^{1 / 3}=\frac{2}{3} \chi \sigma / k T \ln \tilde{s} .
\end{gathered}
$$

Здесь $\chi=\left(6 \sqrt{\pi} M_{C^{*}} /\left(\rho_{l} N_{A}\right)\right)^{2 / 3}, \rho_{l}$ - плотность конденсата, $M_{i}$ - молярные массы компонентов, $N_{A}-$ число Авогадро, $\sigma-$ коэффициент поверхностного натяжения, $\tilde{s}$ - обобщенная степень пересыщения [11]

$$
\tilde{s}=\left(p_{A} p_{B}\right) /\left(p_{A}^{s} p_{B}^{s}\right)
$$

где $p_{i}^{s}$ соответствуют условию равновесия в реакции (4):

$$
\left.\mu_{A}\left(p_{A}^{s}, T\right)+\mu_{B}\left(p_{B}^{s}, T\right)\right)=\mu_{C^{*}}(T) .
$$

В работах $[3,7,12]$ для характеристики метастабильности системы также получены выражения, аналогичные $\tilde{s}$.

4. Для определения константы скорости реакции (5) предположим двухстадийный механизм ее протекания (см. рисунок).

Первой стадией является образование комплексов $Z_{g-1, A}$ или $Z_{g-1, B}$ в соударении молекул $A(B)$ с зародышем $Z_{g-1}$. Второй стадией является собственно химическая реакция между адсорбированной на поверхности зародыша молекулой $A(B)$ и налетающей молекулой $B(A)$. При этом образуются молекулы $C^{*}$ (т.е. зародыш $\left.Z_{g}\right)$. Предполагается, что комплексы $Z_{g-1,2 A}$ или $Z_{g-1,2 B}$, которые также могли бы образоваться

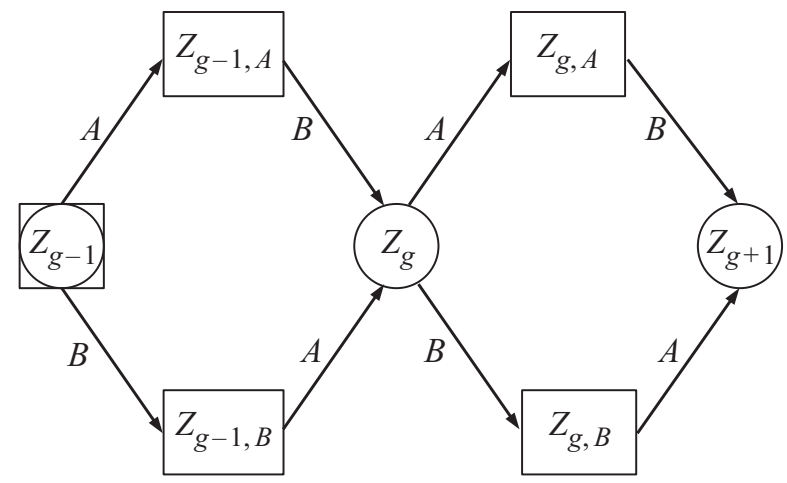

Схема протекания реакции (5).

на второй стадии, нестабильны, и соответствующие процессы их образования не рассматриваются. Использование двухстадийной схемы реакции (5) позволило получить выражение для константы скорости $k(g)$ через константы скоростей двухчастичных процессов первой или второй стадии и по аналогии с подходом Я.Б. Зельдовича [9] получить выражение для скорости стационарной нуклеации в реакции вида (5)

$$
\begin{aligned}
I= & \left(\frac{\alpha_{A} \beta_{A}}{1+\alpha_{A} \beta_{A}\left(\alpha_{B} \beta_{B}\right)^{-1} \Gamma_{A B}}+\frac{\alpha_{B} \beta_{B}}{1+\alpha_{B} \beta_{B}\left(\alpha_{A} \beta_{A}\right)^{-1} \Gamma_{B A}}\right) \\
& \times S\left(g_{c r}\right) \sqrt{\frac{\Delta \Phi\left(g_{c r}\right)}{3 \pi g_{c r}^{2} k T}} N^{0}\left(g_{c r}\right),
\end{aligned}
$$

где $\beta_{i}=p_{i} / \sqrt{2 \pi m_{i} k T}$ - число молекул пара, соударяющихся в единицу времени с единицей поверхности зародыша, $S(g)$ - площадь поверхности зародыша, $\alpha_{i}$ - вероятность прилипания молекулы сорта $i$ при ее соударении с зародышем, $\Gamma_{i j}$ - определяется отношением вероятности прилипания при соударении молекулы сорта $i$ с зародышем на первой стадии к вероятности химической реакции при соударении молекулы сорта $j$ с комплексом на второй стадии.

В случае, когда молекул сорта $B$ в системе значительно больше, чем $A\left(p_{A} \ll p_{B+}, \alpha_{A} \sim \alpha_{B}\right)$, выражение для скорости нуклеации принимает вид

$$
I=\tilde{\alpha}_{A} \beta_{A} S\left(g_{c r}\right) \sqrt{\Delta \Phi\left(g_{c r}\right) / 3 \pi g_{c r}^{2} k T} N^{0}\left(g_{c r}\right),
$$

где $\tilde{\alpha}-$ обобщенный коэффициент конденсации

$$
\tilde{\alpha}_{A}=\alpha_{A}\left(1+\Gamma_{A B} \alpha_{A} \beta_{A} / \alpha_{B} \beta_{B}\right)^{-1} .
$$

В том же приближении для описания регулярного роста сверхкритических зародышей в газокинетическом режиме имеем аналог формулы Кнудсена [13]

$$
\frac{d r}{d t} \equiv \dot{r}=\frac{\tilde{\alpha}_{A}}{\rho_{l}} \sqrt{\frac{M_{C^{*}}^{2}}{2 \pi M_{A} R T}}\left(p_{A}-p_{A}^{s}(r) \sqrt{\frac{T}{T_{d}}}\right) .
$$

Здесь $r$ - радиус зародыша, $m_{i}$ - масса молекулы, $M_{i}$ - молярная масса, $T_{d}$ - температура зародыша. 
5. Результатами данной работы являются выражения, дающие замкнутое описание кинетики объемной конденсации в некоторых сложных системах. Отличие между полученными выражениями (17), (18), (20) и их аналогами для простых систем заключается в следующем. Вопервых, в наличии обобщенного коэффициента конденсации (19) с константой, характеризующей гетерогенную химическую реакцию. Во-вторых, в использовании обобщенной степени пересыщения (15) для вычисления размера, работы образования и равновесной концентрации критических зародышей. В дальнейшем предполагается использование полученных выражений для описания объемной конденсации в конкретных реакциях вида (4).

\section{Финансирование}

Данная работа выполнена при финансовой поддержке Российского фонда фундаментальных исследований (грант № 10-08 00468).

\section{Конфликт интересов}

У автора отсутствует конфликт интересов.

\section{Список литературы}

[1] H. Reiss. J. Chem. Phys. 18, 840 (1950).

[2] J.O. Hirshfelder. J. Chem. Phys. 61, 2690 (1974).

[3] Vitaly V. Slezov. Kinetics of First-order Phase Transitions. WILEY-VCH Verlag GmbH \& Co. KGaA, Weinheim. (2009). $415 \mathrm{p}$.

[4] John H. Seinfeld, Spyros N. Pandis. Atmospheric chemistry and physics: from air pollution to climate change. Wiley, N.Y. (1998). $1326 \mathrm{p}$

[5] Х.Г. Вагнер, К.Ю. Дорге, Д. Танке, П.А. Власов, И.С. Заслонко, А.В. Еремин. Кинетика и катализ 42, 642 (2001).

[6] В.Ю. Киреев, А.А. Столяров. Технологии микроэлектроники. Химическое осаждение из газовой фазы. Техносфера, M. (2006). $190 \mathrm{c}$.

[7] С.А. Кукушкин, А.В. Осипов. ФТТ 50, 1188 (2008).

[8] И.Л. Иосилевский, В.К. Грязнов, А.М. Семенов, Е.С. Якуб, Л.Н. Горохов, В.С. Юнгман, А.Ю. Башарин, М.В. Брыкин, М.А. Шейндлин, В.Е. Фортов. Изв. РАН. Энергетика 5, 115 (2011).

[9] Я.Б. Зельдович. ЖЭТФ 12, 525 (1942).

[10] Я.И. Френкель. Кинетическая теория жидкостей. Наука, Л. (1975). 592 c.

[11] Е.В. Самуйлов, И.Б. Рождественский, Н.М. Корценштейн. Физика и химия обработки материалов 2, 67 (1981).

[12] V.I. Kalikmanov, M.E.H. van Dongen. Phys. Rev. E 55, 1607 (1997).

[13] N.A. Fuchs. Evaporation and Droplet Growth in Gaseous Media. Pergamon Press, N.Y. (1959). 78 p.

Редактор Т.Н. Василевская 